\title{
Digitalsimulator für pulscodierte neuronale Netze
}

\section{H. H. Hellmich and H. Klar}

Technische Universität Berlin, Fakultät für Elektrotechnik und Informatik, Institut für Technische Informatik und Mikroelektronik, Einsteinufer 17, Sekr. EN 4, D-10587 Berlin, Germany

Zusammenfassung. Die Simulation von grossen pulscodierten neuronalen Netzen (PCNNs) für die Evaluierung einer biologisch motivierten Bildverarbeitung ist auf Einprozessor-Systemen (PCs oder Workstations) immer noch sehr zeitineffizient. Den Flaschenhals während der Simulation bildet der sequentielle Zugriff auf den Gewichtsspeicher zur Berechnung der Neuronenzustände. Es wird ein Digitalsimulator basierend auf feld-programmierbaren Gate-Arrays (FPGAs) vorgestellt, der dieses FlaschenhalsProblem durch eine verteilte Speicherarchitektur und eine erhöhte Speicherbandbreite angeht und zusätzlich eine getrennte Berechnung von Neuronenzuständen und Netzwerktopologie vorsieht. Somit ist es möglich, den Parallelitätsgrad des Simulationsalgorithmus zu erhöhen. Die momentane Implementierung mit einer Taktfrequenz von $50 \mathrm{MHz}$ verspricht einen Beschleunigungsfaktor von etwa 30 für eine spärliche Vernetzungsstruktur (Vierer- und Achternachbarschaft) im Vergleich zu einem PC mit einer 2,4 GHz CPU und 1 GB RAM Arbeitsspeicher.

\section{Einleitung}

Die Simulation von PCNNs wird primär aus 2 Gründen durchgeführt. Zum einen möchte man die Pulsverarbeitung von Neuronen im menschlichen Gehirn reproduzieren und verstehen, und zum anderen möchte man die dadurch erlangten Erkenntnisse verwenden, ausgefeilte technische Systeme für unterschiedliche Anwendungsgebiete, insbesondere in der Bildverarbeitung, zu realisieren. Bei der Simulationsbeschleunigung von PCNNs gibt es 5 zu beachtende Flaschenhals-Probleme (Schäfer et al., 2002): Rechenoperationen, Kommunikation, Lastverteilung, Speicherkapazität und Speicherbandbreite. Der Hauptgrund einer ineffizienten Simulationsperformanz bei grossen PCNNs ist die reduzierte Speicherbandbreite (Jahnke et al., 1998), da der Datentransfer zwischen dem Gewichtsspeicher und den ProzessorElementen (PEs) der grösste sequentielle Teil bei der Simulation ist (Reyneri, 2003). Aus diesem Grund ist ein Digitalsimulator für die Simulationsbeschleunigung notwendig, der

Correspondence to: $\mathrm{H}$. H. Hellmich

(hellmich@mikro.ee.tu-berlin.de) dieses grösste Flaschenhals-Problem anhand einer verteilten Speicherarchitektur angeht. Desweiteren stellt der Digitalsimulator eine ausreichende Flexibilität durch programmierbare Software (SW) und rekonfigurierbare BeschleunigungsHardware (HW) anhand von FPGAs bereit.

\section{Neuronenmodell mit adaptiven Synapsen}

Der Digitalsimulator ist primär für das Neuronenmodell von Heitmann und Ramacher (2003) ausgelegt, bei dem es sich um ein Integrate-and-Fire Modell ohne Leckwiderstand handelt. Die Gleichung für das Membranpotental $a_{K}$ ist definiert als,

$a_{K}(t)=a_{K}\left(t_{0}\right)+\int_{t_{0}}^{t}\left[i_{K}+\sum_{i \in N_{S}} W_{K L i}(t)\right] \mathrm{d} t$,

wobei $t_{0}$ der Zeitpunkt des letzten Simulationsereignisses ist, $i_{K}$ repräsentiert den externen Eingangsreiz (Grauwert-Pixel des Eingangsbildes, der auf Werte zwischen 0 und 1 normiert ist), $N_{S}$ ist die Anzahl der Puls-sendenden presynaptischen Neuronen und $W_{K L i}$ stellt den jeweiligen Gewichtswert der Synapse dar. Im Gegensatz zu einem Integrate-and-Fire Neuron mit Leckwiderstand, das nur periodisch feuern kann, wenn das Produkt von konstantem Eingangsstrom und Leckwiderstand grösser als der Schwellwert ist (Gerstner und Kistler, 2002), feuert ein Neuron nach Gl. 1 stets periodisch, wenn ein konstanter Eingangsstrom anliegt. Die neuronale Dynamik des Neuronenmodells basiert auf der Adaption der Synapsengewichte $W_{K L}$ und der Pulsaktivität der presynaptischen Neuronen. Die verschiedenen Adaptionsregeln beeinflussen die zeitliche Ableitung der Synapsengewichte. Die in den Simulationen verwendete Adaptionsregel für die Synapsen lautet wie folgt,

$W_{K L}^{\prime}=-\gamma \cdot W_{K L}+ \begin{cases}\mu \cdot\left(a_{K}-\frac{\theta}{2}\right) & X_{K}=0 \wedge X_{L}=1 \\ 0 & \text { sonst }\end{cases}$

wobei $\gamma$ die Abklingkonstante, $\mu$ den Verstärkungsfaktor und $\theta$ den neuronen-spezifischen konstanten Schwellwert darstellen. Die Variablen $X_{K}$ und $X_{L}$ repräsentieren jeweils den Status des post- bzw. presynaptischen Neurons. Wenn sich das postsynaptische Neuron im Empfangszustand 


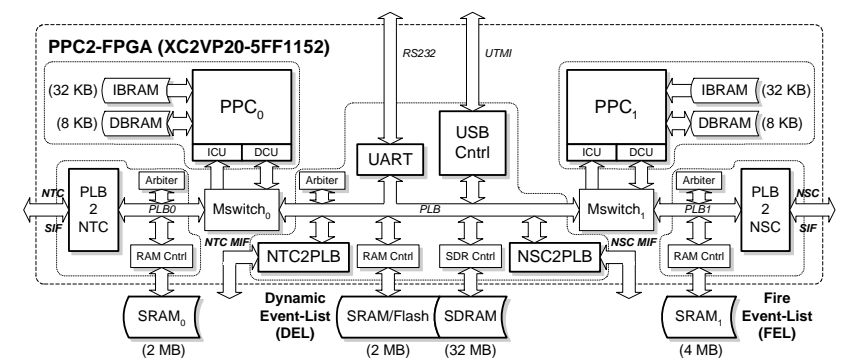

Abbildung 1. FPGA für die Simulationskontrolle.

$\left(X_{K}=0\right)$ und das presynaptische Neuron sich im Sendezustand $\left(X_{L}=1\right)$ befindet, wird das exponentielle Abklingverhalten der Synapse beeinflusst. Abhängig vom Membranpotential des postsynaptischen Neurons findet ein verstärkender Effekt $\left(a_{K}>\theta / 2\right)$ oder ein abschwächender Effekt $\left(a_{K}<\theta / 2\right)$ für die Pulsdauer $t_{d}$ statt. Durch diese neuronale Dynamik werden Neuronen, die von einem ähnlichen externen Eingangsreiz stimuliert werden, zum synchronen Pulsen animiert und können zur Flecken-Detektion einer visuellen Szene eingesetzt werden (Heitmann und Ramacher, 2003).

\section{Architektur des Digitalsimulators}

Die Notwendigkeit für eine neuartige Architektur eines Digitalsimulators beruht auf der Tatsache, dass existierende akademische und kommerziell erhältliche PrototypenPlattformen für die Simulation von grossen PCNNs nicht geeignet sind. Entweder stellen existierende PrototypenPlattformen keine ausreichende Speicherbandbreite und Speicherkapazität zur Verfügung (sog. Rapid-PrototypingPlattformen), oder aber eine ausreichende Speicherbandbreite wird nur in Kombination mit einer Überkapazität an FPGA Ressourcen bereitgestellt (sog. Emulations-Plattformen), die nicht effizient genutzt werden können (Krupnova und Saucier, 2000). Aus diesem Grund stehen bei der Architektur des Digitalsimulators 3 Eigenschaften im Vordergrund: 1. eine kompakte und effiziente Nutzung von FPGA Ressourcen, 2. ausreichende Speicherbandbreite für eine effiziente Parallelisierung des Simulationsalgorithmus, und 3. Autarkie durch ausreichende Speicherkapazität, um den zeitintensiven Datentransfer während der Simulation mit einem Host-Rechner zu vermeiden. Der Digitalsimulator basiert auf 3 FPGAs, die jeweils eigenständige Simulationsaufgaben übernehmen: Simulationskontrolle, Netzwerktopologie- und Neuronenzustandsberechnung (Hellmich und Klar, 2004).

\subsection{Simulationskontrolle}

Die Simulationskontrolle wird vom PPC2-FPGA in Abb. 1 anhand von 2 eingebetteten PowerPCs durchgeführt, die bei Virtex-II-Pro FPGAs bereitgestellt werden (Xilinx, 2004). Die Simulationskontrolle besteht aus 3 Aufgaben: 1. Konfiguration des Netzwerks vor dem Simulationsstart über externe serielle Schnittstellen, 2. Überwachung von

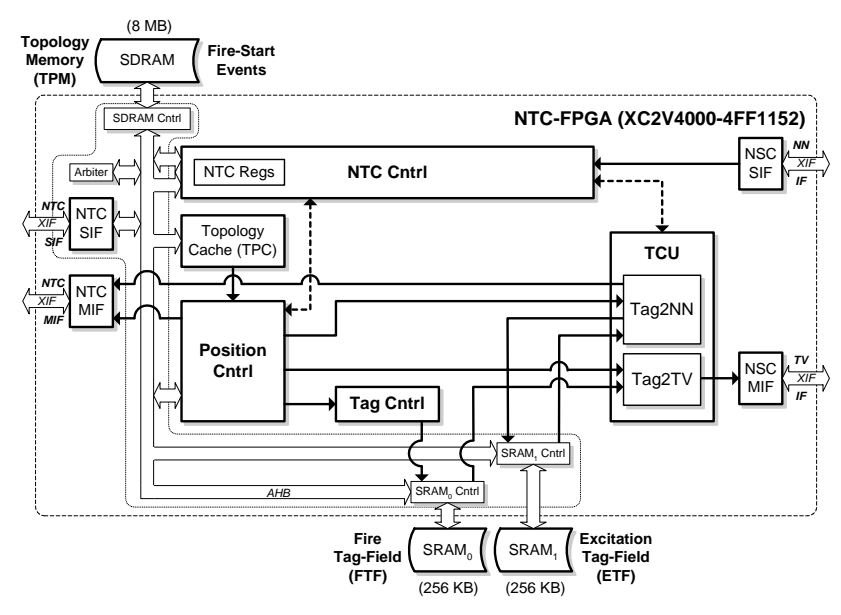

Abbildung 2. FPGA für die Netzwerktopologieberechnung.

Netzwerkparametern, und 3. Verwaltung von Ereignislisten. Während der Simulation müssen 2 Ereignislisten verwaltet werden: die dynamische Ereignisliste (DEL) und die FeuerEreignisliste (FEL). Die DEL beinhaltet alle erregten Neuronen, die zum betrachteten Simulationszeitpunkt einen Puls oder einen externen Reiz empfangen. Die FEL speichert alle Neuronen, die einen Puls senden und sich somit im Sendezustand befinden, und den dazugehörigen Zeitpunkt, an dem das Neuron wieder in den Empfangszustand gelangt. Die maximale Anzahl an Neuronen, die während einer Simulation berücksichtigt werden können, wird von der Speicherkapazität der DEL ( $2 \mathrm{MB}$ ) bestimmt und beträgt $2^{21} / 4=2^{19}$, da ein Neuron mit 4 Bytes codiert wird. Aufgrund des zusätzlich abzuspeichernden Zeitwertes muss die FEL im Vergleich zur DEL eine zweifache Speicherkapazität bereitstellen.

\subsection{Netzwerktopologieberechnung}

Das NTC-FPGA, welches für die Netzwerktopologieberechnung zuständig ist (siehe Abb. 2), operiert in 2 Phasen: Topologie-Vektor-Phase und Topologie-AktualisierungsPhase. Für die Berechnungen sind 2 gleich grosse Markierungsspeicher (Tag-Felder) notwendig: das Feuer-Tag-Feld (FTF), in dem alle feuernden Neuronen markiert sind, und das Erregungs-Tag-Feld (ETF), das alle erregten und feuernden Neuronen markiert.

In der Topologie-Vektor-Phase wird durch Lesen des FTFs die presynaptische Aktivität aller Neuronen bestimmt, die sich in der DEL befinden. Dazu wird ein Bit-Vektor als Topologie-Vektor an die Neuronenzustandsberechnungseinheit gesendet. In der Topologie-Aktualisierungs-Phase wird je nach aufgetretenen Puls-Stop- oder Puls-Start-Ereignis das FTF aktualisiert. Bei Puls-Start-Ereignissen werden durch Lesen des ETFs die postsynaptischen Neuronen bestimmt, die durch den startenden Puls erregt werden und sich noch nicht in der DEL befinden. 


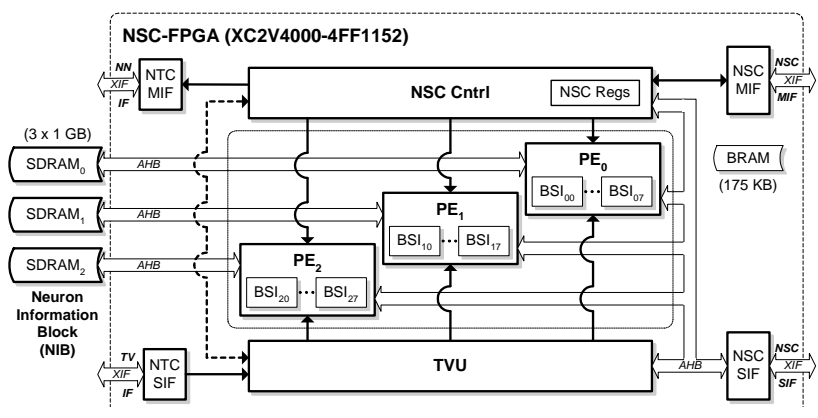

Abbildung 3. FPGA für die Neuronenzustandsberechnung.

\subsection{Neuronenzustandsberechnung}

Die Neuronenzustandsberechnung wird vom NSC-FPGA durchgeführt (siehe Abb. 3). Eine Topologie-Vektor-Einheit (TVU) verteilt dabei die eingehenden Topologie-Vektoren an die 3 vorhandenen PEs. Jede PE beinhaltet mehrere numerische Integratoren (BSIs) und hat einen eigenen Datenkanal zu einem SDRAM Modul, wo NeuronenInformations-Blöcke (NIBs) abgelegt sind. Im Gegensatz $\mathrm{zu}$ anderen Beschleunigungs-Plattformen (Mehrtash et al., 2003) ist somit ein effizienterer Zugriff auf den Gewichtsspeicher möglich, da 3 Datenkanäle parallel genutzt werden können. Ein NIB beinhaltet neben neuronen-spezifischen Parametern, wie dem Membranpotential und dem Schwellwert, alle Synapsengewichte der presynaptischen Neuronen mit synapsen-spezifischen Parametern, wie Abklingkonstanten und Verstärkungsfaktoren. Für jedes Neuron wird ein Informationsblock, der Membranpotential, externen Eingangsreiz und Sprungadresse beinhaltet, von 16 Byte vorgesehen und jeder presynaptischer Gewichtswert, inklusive synapsen-spezifischen Parametern, wird mit 4 Byte codiert. Daraus ergibt sich bei einer Speicherkapazität aller 3 SDRAM Module von insgesamt $3 \mathrm{~GB}$ eine maximale Anzahl von $\left(3 \cdot 2^{30}-2^{19} \cdot 16\right) / 4=803 \cdot 10^{6}$ adaptiven Synpasen, die vom Digitalsimulator berücksichtigt werden können.

Das NSC-FPGA operiert ebenfalls in 2 Phasen: NeuronenPuls-Phase und Neuronen-Aktualisierungs-Phase. Nach dem Lesen der FEL ist der nächste Zeitpunkt bekannt, an dem ein Neuron aufhört zu pulsen (Puls-Stop-Ereignis). In der folgenden Neuronen-Puls-Phase wird ermittelt, ob vor diesem Puls-Stop-Ereignis ein Neuron einen Puls generieren wird (Puls-Start-Ereignis). Dabei muss für alle Neuronen, die Pulse von anderen Neuronen empfangen, nach Gl. 1 eine numerische Integration durchgeführt werden. Bei Neuronen, die lediglich einen externen Eingangsreiz erhalten, lässt sich der Zeitpunkt des Puls-Start-Ereignisses ohne numerische Integration ermitteln $\left(t=\left(\theta-a_{K}\right) / i_{K}\right)$. In der Neuronen-Aktualisierungs-Phase werden schliesslich alle Membranpotentiale und Synpasengewichte mittels der numerischen Integration auf den Zeitpunkt des neu ermittelten Simulationsereignisses aktualisiert. Während eines Simulationsdurchlaufs werden somit maximal 2 numerische Integrationen pro Neuron durchgeführt. Die durchzuführende numerische Integration erfolgt nach dem Bulirsch-Stoer Verfahren (Stoer und Bulirsch, 2000) und besteht im wesentlichen aus 2 arithmetischen Operationen: der ModifiedMidpoint Integration (MMID) und der Polynomextrapolation (PZEXTR). Die MMID berechnet für jeden Funktionswert $y\left(t_{0}\right)$ innerhalb eines Integrationsintervalls $H$ einen neuen Funktionswert $y\left(t_{0}+H\right)$. Dafür werden nstep verschiedene Zwischenwerte berechnet, indem $H$ in gleichgrosse Subintervalle $h=H /$ nstep unterteilt wird. Für die Berechnung des nächsten Zwischenwertes $k_{m+1}$ ist die Ableitung des momentanen Zwischenwertes $k_{m}$ und der Funktionswert des letzten Zwischenwertes $k_{m-1}$ notwendig. Die Formeln für die Berechnung der Zwischenwerte und des neuen Funktionswertes $y_{n s t e p}$ sind der Gl. 3 zu entnehmen,

$$
\begin{aligned}
& k_{m+1}=k_{m-1}+2 \cdot h \cdot f\left(x+m \cdot h, k_{m}\right) \\
& y_{\text {nstep }}=\frac{1}{2} \cdot\left[k_{\text {nstep }}+k_{\text {nstep }-1}+h \cdot f\left(x+H, k_{\text {nstep }}\right)\right]
\end{aligned}
$$

Die PZEXTR führt eine Extrapolation der neu ermittelten Funktionswerte durch, indem nach Gl. 4 die beiden Variablen $Q_{i, k}$ und $D_{i, k}$ berechnet werden,

$$
\begin{aligned}
& y_{i}=Q_{i, 0}=D_{i, 0} ; i=0, \cdots, 7 \\
& Q_{i, k}=\left[y_{i} /\left(y_{i-k}-y_{i}\right)\right] \cdot\left(D_{i, k-1}-Q_{i-1, k-1}\right) \\
& D_{i, k}=\left[y_{i-k} /\left(y_{i-k}-y_{i}\right)\right] \cdot\left(D_{i, k-1}-Q_{i-1, k-1}\right)
\end{aligned}
$$

wobei die Indexvariable $i$ für verschiedene Funktionswerte gilt, die durch die MMID ermittelt wurden. Der extrapolierte Funktionswert $y_{\text {ext }, i}$ ergibt sich dann aus der Summe nach Gl. 5,

$y_{\text {ext }, i}=\sum_{k=0}^{i} Q_{i, k}$

\section{Abschätzung der Performanz}

Für die Leistungsabschätzung des Digitalsimulators wurden 5 Eingangsschichten mit unterschiedlicher Neuronenanzahl $(32.32 ; \ldots$; 96.96) simuliert. Die Neuronen dieser Schichten sind lateral im Rahmen einer Vierernachbarschaft $(n=4)$ oder einer Achternachbarschaft $(n=8)$ miteinander verbunden. Jede Simulation wurde bis zu einer Netzwerkzeit von $500 \mathrm{~ms}$ durchgeführt. Die Membranpotentiale aller Neuronen wurden mit zufälligen Werten zwischen 0 und 1 inititalisiert $\left(\theta=1 ; t_{d}=1 \mathrm{~ms}\right)$ und für alle Synapsen, die mit dem Wert 0,12 initialisiert wurden, wurde die Adaptionsregel nach Gl. 2 angewendet $(\gamma=0,1 ; \mu=0,3)$.

\subsection{Software Simulationszeiten}

Während der SW Simulationen, die auf einem Linux-PC (2,4 GHz Pentium-4; 1 GB RAM) durchgeführt wurden, wurden 4 Variablen protokolliert: die Anzahl der aufgetretenen Simulationsereigniszeiten $\left(N_{E V E N T}\right)$, die Anzahl der durchgeführten Integrationen pro Neuron $\left(N_{B S S T E P}\right)$, die Anzahl der Integrationsintervalländerungen $\left(N_{H}\right)$ und die Anzahl der notwendigen Integrationsschrittunterteilungen pro Integration $\left(N_{I}\right)$. In Abb. 4 sind diese Variablen für die 




Abbildung 4. Durchgeführte Integrationen pro Neuron.

5 Eingangsschichten mit einer Vierernachbarschaftsverbindung dargestellt. Man erkennt, dass für jede durchgeführte Integration nahezu keine Integrationsintervalländerung notwendig war $\left(H_{A V G}=N_{H} / N_{B S S T E P} \simeq 1\right)$. Die Betrachtung der Variablen $N_{I}$ verdeutlicht, dass durchschnittlich 2 Integrationsschrittunterteilungen pro Integration notwendig waren $\left(I_{A V G}=N_{I} / N_{B S S T E P} \simeq 2\right)$. Die SW Simulationszeiten $T_{S W}$ berücksichtigen nur die Zeit, die für die Durchführung der numerischen Integration notwendig war (etwa 70\% der gesamten Simulationszeit). In Abb. 5 sind die SW Simulationszeiten, die mit zunehmender Anzahl der Neuronen $\left(N_{N E U R O N}\right)$ und Synapsen $\left(N_{W E I G H T}\right)$ exponentiell ansteigen, dargestellt. Die Simulation einer 96.96 grossen Eingangsschicht mit einer Achternachbarschaftsverbindung dauert somit mehr als anderthalb Tage (142 $834 \mathrm{~s}$ ).

\subsection{Simulationszeiten des Digitalsimulators}

Die Simulationszeiten des Digitalsimulators $T_{D S}$ lassen sich nach folgender Formel abschätzen,

$T_{D S}=N_{B S S T E P} \cdot \frac{N_{N E U R O N}}{N_{I O}} \cdot T_{C L K} \cdot T_{N E U R O N}(n)$

wobei $N_{N E U R O N}$ der Anzahl der zu simulierenden Neuronen entspricht, $N_{I O}$ ist die Anzahl der parallel nutzbaren Datenkanäle, $T_{C L K}$ ist die Taktperiode und $T_{N E U R O N}$ entspricht der Taktanzahl, die für die numerische Integration eines Neurons benötigt wird. Diese Taktanzahl ist von der Anzahl $n$ der presynaptischen Gewichte abhängig und kann wie folgt berechnet werden,

$$
\begin{aligned}
T_{N E U R O N}= & H_{A V G} \cdot \sum_{i=0}^{I_{A V G}-1}\left[T_{M M I D}(n, i)\right. \\
& \left.+T_{P Z E X T R}(n, i)\right]+t_{S D R A M}
\end{aligned}
$$

wobei $t_{S D R A M}$ die Latenzzeit in Takten für den Zugriff der NIBs im SDRAM ist. $T_{M M I D}$ und $T_{P Z E X T R}$ stellen die Anzahl der Takte dar, die für die MMID und die PZEXTR notwendig sind. Die notwendige Taktanzahl für die MMID ist in Gl. 8 angegeben,

$\left.T_{M M I D}=t_{M M I D}+\operatorname{ceil}(n / 2) \cdot[2 \cdot(i+1)+1)\right]$



Abbildung 5. Synapsen, Neuronen und Software Simulationszeiten.

wobei $t_{M M I D}$ der Latenzzeit in Takten entspricht, bevor das erste gültige Datum aus der Pipeline des implementierten HW Moduls erscheint. Der Term $n / 2$ in Gl. 8 kommt dadurch zustande, dass die Datenbusbreite der SDRAM Module 8 Bytes beträgt und somit stets 2 Synapsengewichte gleichzeitig gelesen werden können. Die notwendige Taktanzahl für die PZEXTR ist in Gl. 9 beschrieben,

$$
\begin{aligned}
T_{P Z E X T R}= & t_{P Z E X T R}+\operatorname{ceil}(n / 2) \\
& +i \cdot\left[\operatorname{ceil}\left(n /\left(2 \cdot t_{P Z E X T R}\right)\right) \cdot t_{P Z E X T R}\right]
\end{aligned}
$$

wobei $t_{P Z E X T R}$ wieder der Latenzzeit in Takten entspricht, die aufgrund der Pipeline des HW Moduls resultiert. Anhand Gl. 8 und Gl. 9 kann nun durch Einsetzen der entsprechenden Latenzzeiten $\left(t_{S D R A M}=10, t_{M M I D}=12, t_{P Z E X T R}=4\right)$ die Berechnungsdauer eines Neurons in Takten mittels Gl. 7 für eine Vierer- $(n=4)$ und Achternachbarschaft $(n=8)$ angegeben werden $\left(H_{A V G}=1, I_{A V G}=2\right)$,

$$
T_{\text {NEURON }}(n=4)=66 \quad T_{N E U R O N}(n=8)=86
$$

Für eine Taktfrequenz von $50 \mathrm{MHz}\left(T_{C L K}=20 \mathrm{~ns}\right)$ und der parallelen Nutzung von 3 Datenkanälen $\left(N_{I O}=3\right)$ lassen sich mit der durch die SW Simulation ermittelten Anzahl an durchgeführten Integrationen pro Neuron $\left(N_{B S S T E P}\right)$ die Simulationszeiten des Digitalsimulators mit Hilfe von Gl. 6 abschätzen. Die resultierenden Beschleunigungsfaktoren $F$ berechnen sich aus dem Verhältnis von $T_{S W} \mathrm{zu} T_{D S}$ und sind in der Tabelle 1 zusammengefasst.

\section{FPGA Prototypen-Platine}

Die realisierte FPGA Prototypen-Platine in Abb. 6 zeigt die 3 FPGAs, auf denen die Architektur des Digitalsimulators beruht. Das PPC2-FPGA hat Zugang zu den beiden Ereignislisten DEL und FEL, das NTC-FPGA hat Zugriff auf die beiden Tag-Felder FTF und ETF, und das NSC-FPGA steuert die 3 SDRAM Module an, um die entsprechenden NIBs aktualisieren zu können. Die $250 \times 150 \times 2 \mathrm{~mm}$ grosse 10-lagige 
Tabelle 1. Abgeschätzte Simulationszeiten des Digitalsimulators.

\begin{tabular}{|c|c|c|c|c|c|}
\hline$n$ & $N_{N E U R O N}$ & $N_{B S S T E P}$ & $T_{S W}$ & $T_{D S}$ & $F$ \\
\hline & $32 \cdot 32$ & 98717 & $1405 \mathrm{~s}$ & $45 \mathrm{~s}$ & 31,2 \\
\hline & $48 \cdot 48$ & 222365 & $6527 \mathrm{~s}$ & $226 s$ & 28,9 \\
\hline & 64.64 & 420299 & $22620 \mathrm{~s}$ & $758 \mathrm{~s}$ & 29,8 \\
\hline & $80 \cdot 80$ & 721463 & $65277 \mathrm{~s}$ & $2032 \mathrm{~s}$ & 32,1 \\
\hline & $96 \cdot 96$ & 926458 & $119109 \mathrm{~s}$ & $3757 \mathrm{~s}$ & 31,7 \\
\hline & $32 \cdot 32$ & 107276 & $1990 \mathrm{~s}$ & $63 \mathrm{~s}$ & 31,6 \\
\hline & $48 \cdot 48$ & 235863 & $7263 \mathrm{~s}$ & $312 \mathrm{~s}$ & 23,3 \\
\hline & 64.64 & 413861 & $31548 \mathrm{~s}$ & $972 \mathrm{~s}$ & 32,5 \\
\hline & $80 \cdot 80$ & 645694 & $80378 \mathrm{~s}$ & $2370 \mathrm{~s}$ & 33,9 \\
\hline & $96 \cdot 96$ & 967572 & $142834 \mathrm{~s}$ & $5113 \mathrm{~s}$ & 27,9 \\
\hline
\end{tabular}

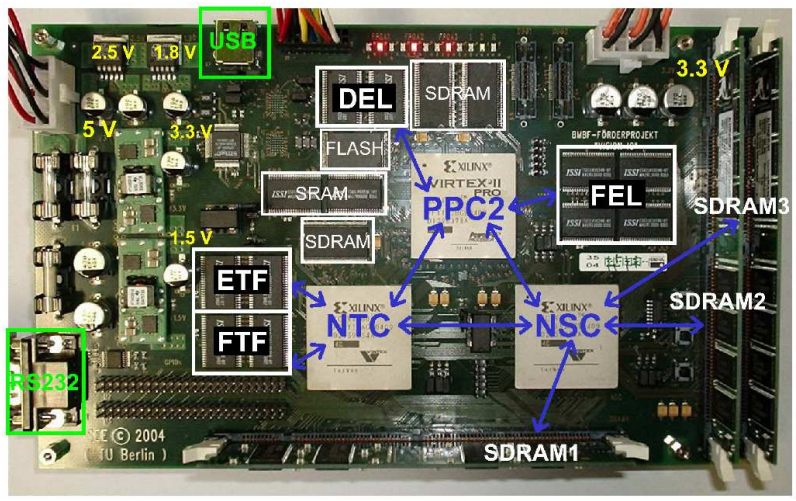

Abbildung 6. FPGA-Prototypen-Platine des Digitalsimulators.

Prototypen-Platine (2 Masse-, 2 Versorgungs- und 6 SignalLagen) verwendet mehr als 350 Entkoppelkapazitäten und muss 5 verschiedene Versorgungsspannungen $(1.5 \mathrm{~V} ; 1.8 \mathrm{~V}$; $2.5 \mathrm{~V} ; 3.3 \mathrm{~V}$ und $5 \mathrm{~V}$ ) bereitstellen.

\section{Schlussfolgerungen}

Es wurde eine Digitalsimulator für grosse PCNNs vorgestellt, dessen Flexibilität auf der Programmierbarkeit von SW und auf der Rekonfigurierbarkeit von beschleunigender HW basiert. Bei einer Taktfrequenz von $50 \mathrm{MHz}$ verspricht der Digitalsimulator einen Beschleunigungsfaktor von etwa 30 für eine spärliche Vernetzungsstruktur (Vierer- und Achternachbarschaft) im Vergleich zu einem konventionellen PC. Die Architektur des Digitalsimulators ist durch eine verteilte Speicherarchitektur, eine effiziente FPGA-Nutzung, eine hohe Speicherbandbreite und ausreichende Speicherkapazität charakterisiert. Die realisierte FPGA-Prototypen-Platine befindet sich zur Zeit in der Testphase, um die Funktionalität des Digitalsimulators zu überprüfen und die abgeschätzten Simulationszeiten zu validieren.

\section{Danksagung}

Wir danken W. Maurer und D. Backhaus von der CAD-UL $\mathrm{GmbH}$ für die Unterstützung bei der Entflechtung und Bestückung, sowie der Rinde GmbH für die Herstellung der Prototypen-Platine.

\section{Literatur}

Gerstner, W. und Kistler, W. M.: Spiking Neuron Models - Single Neurons, Populations, Plasticity, Cambridge University Press, ISBN 0-521-81384-0, 2002.

Heittmann, A. und Ramacher, U.: Correlation-Based Feature Detection Using Pulsed Neural Networks, IEEE 13th Workshop on Neural Networks for Signal Processing, 479-488, 2003.

Hellmich, H. H. und Klar, H.: An FPGA based Simulation Acceleration Platform for Spiking Neural Networks, IEEE 47th Midwest Symposium on Circuits and Systems, 2, 389-392, 2004.

Jahnke, A., Roth, U. und Schönauer, T.: Digital Simulation of Spiking Neural Networks, Pulsed Neural Networks, Herausgeber: Maass, W. und Bishop, C. M., MIT Press, ISBN 0-262-13350-4, 1998.

Krupnova, H. und Saucier, G.: FPGA-Based Emulation: Industrial and Custom Prototyping Solutions, 10th Int. Conf. on Field Programmable Logic and Applications, 68-77, 2000.

Mehrtash, N., Jung, D., Hellmich, H. H., Schönauer, T., Lu, V. T. und Klar, H.: Synaptic Plasticity in Spiking Neural Networks (SPINN): A System Approach, IEEE Trans. on Neural Networks, 14-5, 980-992, 2003.

Reyneri, L. M.: Implementation Issues of Neuro-Fuzzy Hardware: Going Toward HW/SW Codesign, IEEE Trans. on Neural Networks, 14-1, 176-194, 2003.

Schäfer, M., Schönauer, T., Wolff, C.,Hartmann, G., Klar, H. und Rückert, U.: Simulation of Spiking Neural Networks: Architectures and Implementations, Neurocomputing, 48, 647-679, 2002.

Stoer, J. und Bulirsch, R.: Numerische Mathematik II, Springer Verlag, ISBN 3-540-67644-9, 2000.

Xilinx: Virtex-II/II-Pro Datenblätter, www.xilinx.com/xlnx/xweb/ xil_publications_index.jsp, 2004. 